\title{
Tránsito de la fenomenología a la hermenéutica. Heidegger lector de Confesiones X, 1-23
}

\section{Transit from Phenomenology to Hermeneutics. Heidegger reader of St. Augustine's Confessions X, 1-23}

\author{
Claudio César Calabresse \\ Universidad Panamericana, México \\ ccalabrese@up.edu.mx
}

Recibido: 16/11/2015 • Aceptado: 01/09/2016

\begin{abstract}
Resumen
En este artículo, hacemos un seguimiento de la lectura que hiciera el joven Heidegger del libro X (capítulos 1-23) de Las Confesiones; en ella se presenta, por un lado, la refutación de aquellos planteamientos que intentan alcanzar síntesis de épocas y que por ello desrealizan necesariamente el ahora y el yo de Agustín, en este caso. Luego seguimos de cerca los distintos pasos del procedimiento de lectura de Heidegger y sus resultados.
\end{abstract}

Palabras clave: Agustín de Hipona, agustinismo, fenomenología, hermenéutica, joven Heidegger.

Abstract

In this paper, we track the young Heidegger's reading on Book X (chapters 1-23) of Confessions; it shows, on the one hand, the refutation of those positions that seek to achieve a synthesis of different epochs and that, therefore, necessarily draw out of reality Augustine's «now» and his «self» in this case. Then, we follow closely the various steps of Hidegger's process of reading and its results.

Keywords: Augustine of Hippo, Augustinism, Hermeneutics, Phenomenology , Young Heidegger. 
Introducción

En el presente trabajo, se considera la siguiente tesis: en la lectura del libro X de las Confesiones (capítulos 1-23), ${ }^{1}$ Heidegger define el tránsito de la fenomenología a la hermenéutica. En este paso, en el que se despliegan las tensiones entre «objetivo» y «subjetivo» y la imposibilidad de una elección unilateral de uno u otro, se pone de manifiesto la importancia de la noción del «no-camino» (die Umbeg), uno de los modos de estar en relación con el mundo.

La argumentación en favor de la tesis tiene dos momentos: por un lado, presentamos el desmonte que efectúa Heidegger de la bibliografía más representativa de la época, en lengua alemana, dedicada a la comprensión de las obras y a la significación histórica de san Agustín; de este análisis hacemos un seguimiento literal, porque prepara y delimita la lectura propiamente dicha de san Agustín y los modos de su realización. Por otro lado, presentamos la experiencia de lectura de Heidegger y sus claves.

Si bien la mencionada lectura conlleva instancias de apropiación (tal como presentamos más adelante), en nuestro artículo nos detenemos en los intersticios que va dejando Heidegger cuando cita a san Agustín e introduce -entre paréntesis- un comentario o una glosa, o cuando el propio Heidegger realiza una síntesis ante el frecuente recurso estilístico de la amplificación.

Heidegger plantea su lectura como deslinde (Heidegger, 1995: 159), respecto de la particular concepción histórica y doctrinal de Ernst Troeltsch, Adolf von Harnack y Wilhelm Dilthey; todas ellas concuerdan en la alta valoración del papel asignado a san Agustín. Coinciden en que en él descansa la teología medieval y en que su recepción se dio como agustinismo o platonismo cristiano, es decir,

1 En lo sucesivo, se abreviará: Conf. 
como el entramado de interpretaciones de que fue objeto la obra de san Agustín a lo largo de la Edad Media, hasta el siglo XIII y, desde ahí, en intensa discusión con el aristotelismo, como una determinada concepción de la gracia. Estos autores comparten, aunque en distinto grado, el intento de objetivar el pensamiento de san Agustín, lo que trajo como consecuencia impedir o, al menos, dificultar lecturas renovadas.

En el segundo punto, el mencionado seguimiento de los distintos pasos de la lectura implica distinguir tres instancias: a) avanzar con el procedimiento de «la ejecución del acceso» (Zugangsvollzug); b) «el sentido de acceso» (Zugangssinn); c) «el saber gozoso» (Gaudium de veritate).

Este artículo pretende, entonces, seguir el rastro de una lectura de san Agustín original y profunda, ordenada por una intuición en la que el propio Heidegger anticipa aspectos de la metodología de Ser y Tiempo.

Los momentos previos de la interpretación: el triple deslinde de Heidegger

El texto de Ernst Troeltsch estudia a san Agustín desde el punto de vista de la filosofía de la cultura. "El problema de la cultura pasó a convertirse en el gran problema de los pensadores cristianos", cita Heidegger a Troeltsch (1995: 160); para Heidegger, «el problema de la cultura» (das Kulturproblem) significa el modo en que el cristianismo asume los bienes de la cultura en el contexto de la noción de salvación.

El carácter acuciante del problema radica en que la respuesta implica la forma en la que el cristiano, individualmente, y la cristiandad, como paradigma, resuelven su relación con el mundo, dado que la propia noción de trascendencia había abierto una grieta en el corazón mismo del paganismo, pues éste, en el marco de sus grandes corrientes filosóficas, enfatiza la mutua dependencia entre cosmosBien con Platón, cosmos-Primer Motor con Aristóteles y cosmosUno con Plotino.

Heidegger alerta que la trascendencia teórica tuvo como correlato la declinación del valor de la experiencia (Flamarique, 2013: 
116-117). La filosofía de la cultura será para el cristianismo, al menos a partir de san Agustín, el intento permanente de reconciliarse con el mundo como arquitectura del saber y como fuente de sabiduría.

Heidegger descalifica la interpretación de Troeltsch, pues este último entiende el problema de la cultura, en primer término, como orientación en la formación educativa y luego como expresión de una dogmática teológica; en efecto, este modo de comprensión no deja espacio para la interpretación fáctica, que es del interés de Heidegger; por ello, señala que su exposición tiene el defecto de la generalización (Heidegger, 1995: 162).

El segundo texto que revisa Heidegger es el de Adolf von Harnack: a su juicio, este autor tiene mayor familiaridad con los textos de san Agustín que Troeltsch y su modo de enfrentar el tema es más conciso: desde la historia de los dogmas.

Es evidente que el enfoque es más atractivo para el empeño de Heidegger, pues von Harnack percibe que lo más característico de san Agustín no consistió en configurar un nuevo sistema dogmático, sino en renovar la religiosidad mediante la piedad, es decir, puso la religiosidad en el centro de la comunidad y el cultivo de los corazones como don y tarea.

La noción de renovación de un sistema dogmático mediante la gracia concita el interés de Heidegger, en cuanto conlleva la tensión entre tarea del teólogo y existencia en la gracia, cuyo cometido es renovar la comprensión del mundo en el propio corazón y en la comunidad que lo vive (Caputo, 1999: 284).

La lectura de Heidegger se interesa en este paso, en cuanto lo interpreta en los términos de una religiosidad que busca vivir el dogma en el sujeto, pues en él se expresa el impulso hacia la plenitud de la auto-comprensión (Camilleri, 2008: 242).

En la visión de W. Dilthey, el cristianismo implica una notable novedad: la vida anímica se concentra en sí misma. ¿En qué consiste la vida nueva que trae el cristianismo? La ciencia antigua se había mantenido en los límites del mundo externo, pero ahora la vida anímica se convierte en problema (Stanley, 2010: 71-73).

Para Heidegger, la consecuencia central es que la revelación de Dios en Cristo (realidad histórica) sale de la trascendencia de claro 
cuño platónico e ingresa de lleno en la experiencia (Heidegger, 1995 : 164). Éste, para Dilthey, es el origen de la conciencia histórica.

Para el mismo autor, lo más significativo del aporte de san Agustín es que opuso la realidad de la experiencia interior al escepticismo. Pero este aporte queda, en buena medida, invalidado por el giro hacia la metafísica (en sentido clásico, que para Heidegger significa «platónico»): «las verdades eternas» son las ideas en la conciencia absoluta de Dios.

Para Dilthey, la búsqueda de Agustín se centra en hallar un fundamento por el cual el alma permanezca idéntica a sí misma y así sostenga y explique sus transformaciones; Heidegger rechaza completamente la afirmación que de aquí deriva Dilthey: esto no se logró hasta Kant y Schleiermacher.

La crítica a W. Dilthey conlleva plantear el problema de la objetividad histórica como condición de posibilidad de la conciencia histórica pues se trata, precisamente, de su formación. Aunque interpretándola a contraluz, la lectura de Dilthey ha resultado decisiva para Heidegger.

La pregunta acerca de cuál de las tres concepciones históricas de aproximación a san Agustín -cultural, dogmática o científica- sea la correcta desnaturaliza el planteo de la objetividad histórica. En efecto, no es posible inferir interpretativamente la concepción usual y acrítica de verdadero y falso en la historia (Heidegger, 1995: 165). Ello implica tanto desechar el relativismo que surge de la simple comparación de las distintas visiones y del escepticismo que se encuentra en su planteo. Dicho escepticismo se haría también presente en la decisión de declarar igualmente válidas las tres aproximaciones.

Se comprende así que el ordenamiento cronológico de los hechos acontecidos en el pasado no implica necesariamente la comprensión historiográfica de ellos: ¿cuál es la medida de los conocimientos heredados? La consecuencia inmediata de esta pregunta requiere fundamentar la objetividad histórica en la comprensión del despliegue de la conciencia histórica (Escudero, 2008: 52).

La decisión de Heidegger de no entregar las nociones de experiencia y conocimientos históricos a la mensura del conocimiento heredado le obliga a plantear su apropiación genuina; ello implica 
la apertura del "sentido de la ejecución de la experiencia histórica operante” (Heidegger, 1995: 166).

Procedimiento que desmonta las lecturas de Troeltsch, von Harnack y Dilthey

Debemos preguntarnos sobre el alcance del término «ejecución» [der Vollzug], en el texto, según la acepción que se emplea para las obras musicales. Heidegger retoma este término, que proviene de la herencia fenomenológica de Husserl, ${ }^{2}$ en quien representa el carácter realizativo de cada acto de conciencia (Heidegger, 1995: 274297).

El primer paso de la fenomenología como hermenéutica consiste en considerar primero «el sentido del acceso» (Zugangssinn) y luego «la base motivacional para el enfoque y la ejecución del acceso» (Motivbasis für Zugangsansatz und Zugangsvollzug).

Si bien las «direcciones del acceso» (Zugangsrichtungen) son diversas (ética, religiosidad, fundamentación teórica), concuerdan en el sentido de ese acceso, es decir, la lectura de san Agustín se realiza desde los tres puntos de vista, cada uno de los cuales ordena su interpretación en un marco diverso.

La captación de las estructuras del ser es el objeto de la aplicación del modelo de lectura de Heidegger; la condición de posibilidad de alcanzar este objetivo radica en la capacidad de distinguir las categorías conceptuales que constituyen las diversas interpretaciones culturales y experienciales.

«La ejecución del acceso» (Zugangsvollzug) consiste en echar raíces en la vida; «ejecución» (derVollzug), en efecto, es el punto de partida de la comprensión; ello implica que la realidad es resultado de

2 En E. Husserl, en la relación Vollzug - Vorliegen («efecto» - «efectuación»), el sujeto sale para captar el objeto en su propia identidad (la identidad que le es propia en cuanto objeto), en el sentido de que toda efectuación, en tanto proceso intencional, se orienta teleológicamente al objeto. Vollzug entraña la noción de «efecto», pues se encuentra en el núcleo propositivo de aquella identidad. (Held, 1995: Il, 274-297; Toadvine, 2002: 282-286). 
aquella efectuación y que tiene un claro sentido semántico, es decir, un volver a la co-pertenencia originaria del lenguaje y del ser, en el horizonte del interrogar la vida.

Se termina de definir aquí el hiato con la metafísica tradicional u objetiva: ésta descansa en la seguridad de poder descubrir lo constitutivo de la realidad mediante el lenguaje. Por el contrario, en el sentido que Heidegger plantea «ejecución» (derVollzug) el obrar está sostenido en la semántica que el proceso implica. Esto significa, por un lado, que, en el caso de san Agustín, el acceso a la vida fáctica es textual y, por otro, que la puesta en marcha o ejecución del proceso consiste en interpretaciones que organizan un horizonte de sentido, pues no otra cosa implica establecer la vida fáctica en sí.

Para Heidegger, entonces, al ubicar Troeltsch a Agustín en el centro de la evolución cultural de la Antigüedad, su figura queda objetivada en la fijación de la ética cristiana; en este punto queda establecido su significado universal, en cuanto a la persona y en cuanto a sus obras y, a partir de allí, todo el marco interpretativo (Stanley, 2010: 77).

Se confronta así que lo esencial está dado por el marco filosófico-cultural: lo máximamente abarcador y, por ende, lo más diluido y escurridizo al intento de interpretación; la universalidad, que caracteriza el intento de Troeltsch, es un modo de coacción para la comprensión de la vida histórica genuina.

La opción historiográfica objetivante aspira a contener un determinado logro filosófico en estructuras necesariamente abstractas, que se colocan por encima de la consideración del trabajo mediante el cual se alcanzó aquel logro. El cierre de Heidegger es contundente: estudios de esta naturaleza encubren "una aguada reelaboración de bibliografía secundaria” (Heidegger, 1995: 167).

Alcanzar la vitalidad de las concepciones filosóficas implica encontrarse con formas previas de la experiencia del pensamiento, las que suelen pasar desapercibidas en los grandes textos. Se delimita así el campo donde no es posible la facticidad; se alerta sobre el riesgo permanente de que las estructuras teóricas tergiversen la experiencia, en la profundidad que ésta tenga. Por ello, la correlación entre vida fáctica, lenguaje y ser como esfera de copertenencia es un 
recurso para poner a salvo la relación subyacente entre la vida como se vive y lo que permanece como experiencia en el sujeto (Campbell, 2012: 23 ss.).

La convicción de Troeltsch, la que se encuentra oculta tras sus frases y su vocabulario, busca rendir un servicio a la vida religiosa de su tiempo, al ofrecer una orientación general a través del seguimiento sobre cómo se han fijado lo que llamamos «valores culturales».

Luego, san Agustín como re-formador, entendiendo el término como la fijación del camino que toman las definiciones dogmáticas (von Harnack). Aquí la dirección del acceso es más acotada y, por lo tanto, permite una visión de san Agustín más finamente calibrada, en tanto podemos seguir sus pasos, su herencia intelectual, como Doctor de la gracia. Continúa, a pesar de ello, en el contexto que Heidegger ha especificado como objetivante. La convicción de von Harnack (Stanley, 2010: 6-7), teólogo luterano que marcó el tránsito del siglo XIX al XX con la idea de que el Evangelio se había desnaturalizado con la helenización operada por los teólogos del s. II, muestra la distancia que existe entre lo que Heidegger llama teología eclesial (la actual a su momento) y la orientación de la teología cristiano-protestante.

Por último, despacha a Dilthey en dos líneas, que tienen la misma dirección de lo dicho para von Harnack, pero hacia el desarrollo de las ciencias del espíritu: "Lo mismo vale para Dilthey" (Heidegger, 1995: 166).

Si bien las perspectivas analizadas carecen, para Heidegger, de un valor positivo en el avance de la investigación, en tanto que una reflexión histórico-objetivante no posibilita la comprensión efectiva y directa de lo estudiado, por el contrario sí permite, como envés de la misma trama, comprender que aquella investigación no puede estar desgajada de la vida.

Todas las dificultades de este intento quedan claramente expuestas en la superficie, pues el método fenomenológico mismo se resquebraja, si en su operar no emerge y se hace evidente la vida. Esta limitación de los procesos de objetivación no implica la solución simple de recorrer las posibilidades de un punto de vista subjetivo. 
Una oposición de esta naturaleza como hipótesis de trabajo carece de sentido, pues «objetivación» y «punto de vista subjetivo» caminan una junto a la otra alejándose al mismo tiempo del objeto.

Desde el título de la obra, Agustín y el Neoplatonismo, tenemos clara su veta histórico-objetiva: la recepción de conceptos y términos del neoplatonismo, es decir: la materia y el medio que san Agustín fundió, en sus vertientes tanto filosófica cuanto teológica. Ésta resulta la trama visible de aquellos procesos en los cuales echa raíces el mundo que aún hoy vivimos o, en palabras de Heidegger, "el problema general" (Heidegger, 1995: 167).

Pero, si sólo ésta fuese la perspectiva, la que, en definitiva, observamos en los estudios anteriores, quedaríamos en los límites del problema tal como se planteaba en la década de los '20 y de los '30 del siglo pasado, deudora -a su vez- de los estudios históricodogmáticos de teólogos liberales del siglo XIX de la talla de Albrecht Ritschl, es decir, la relación entre Grecia y el cristianismo.

Aun aceptando que el cristianismo no se heleniza con san Agustín, sino que el Hiponense respira una atmósfera que había comenzado este proceso desde el s. II, el Neoplatonismo ofrecía las herramientas necesarias para una exposición de los contenidos de la fe.

Éste es el punto para Heidegger, pues la lectura de estos procesos desde una perspectiva histórico-objetiva o de reconstrucción vivencial (la vivencia como medio de conocimiento psicológico) conllevan la imposibilidad de comprender la «vida fáctica»; aplicado este giro a san Agustín, la fe ocupa el lugar central y debe ser presentada en términos de experiencia, es decir, en la fe misma y no en un puro sistema racional de referencias (Heidegger, 1995: 171-172).

Los momentos de la interpretación: la lectura de Conf. X, 1-23.

La interpretación tiene como punto de partida la lectura en clave hermenéutica del pasaje de Retractationes dedicado a Confessiones. Luego, lo que Heidegger considera la introducción al Libro X (capítulos primero a séptimo), es decir, los sentidos del confesar (confiteri) ante Dios y ante los hombres. Los capítulos octavo al decimonoveno 
se ocupan del tema central de la memoria (quién soy para mí en los jalones de mis recuerdos y olvidos). Las consideraciones sobre la vida feliz [vita beata], a lo largo de los capítulos vigésimo a vigesimotercero, y su vínculo con el gozo de la verdad [Gaudium de veritate].

Las Retractationes ${ }^{3}$ implican para san Agustín una revisión o relectura que salve la unidad doctrinaria a lo largo de las distintas etapas de su vida intelectual: "corrijo mis errores" (Retr. 1). Este aspecto, que podría formar parte de la historia de los dogmas, en la vertiente objetivante señalada por Heidegger, no entra en el ámbito de la vida pues, puesta en contexto, se refiere al conjunto de sus escritos.

El examen se detiene en el parágrafo siguiente, en el que san Agustín dará dos notas susceptibles del análisis existencial que Heidegger considera conveniente para el prólogo: por un lado, la cita del texto bíblico de Proverbios X, 19 ("En el mucho hablar no faltará el pecado") y, por otro, la turbación profunda (Retr. 1) que el Hiponense experimenta ante sus escritos; y esto en un doble sentido: ante sus propios errores, que lo llevan a ocuparse de estas recensiones, y por los escritos que circulan a su nombre, pero que no han salido de su pluma, que, entonces, no forman parte de su entramado vital.

De los pasajes de Retractationes dedicados a Confessiones cita (Heidegger, 1995: 175):

Los trece libros de mis Confesiones alaban a Dios, justo y bueno, tanto por mis obras malas como por las buenas, y mueven hacia él el espíritu y el corazón humanos. Al menos en cuanto a mí, eso hicieron en mí cuando las escribí, y continúan haciéndolo cuando se leen. Qué piensan otros de ellas, allá ellos; sin embargo, sé que a muchos hermanos les han gustado mucho y continúan gustándoles. Tratan de mí desde el libro primero hasta el décimo; los tres restantes tratan de las Sagradas Escrituras, desde aquello "En principio Dios creó el cielo y la tierra", hasta el descanso sabático (Gen. 21, 2). ${ }^{4}$

3 En lo sucesivo, Retr.

4 Retr., 2: "Confessionum mearum libri tredecim, et de malis et de bonis meis Deum laudant iustum et bonum, atque in eum excitant humanum intellectum et affectum. In- 
Heidegger no traduce a Agustín, pero introduce glosas que nos orientan en la dirección de su interpretación. "Los trece libros de mis Confesiones, tanto por mis obras malas como por las buenas [en mi ser, en mi vivir, en mi haber sido malas y buenas], alaban a Dios justo y bueno...” (Heidegger, 1995: 175-176). ${ }^{5}$

Veamos, entonces, cuál es la dirección de la comprensión, sus medios y los términos de la ejecución de la comprensión. El comentario o la exposición [das Referat] no es un nuevo texto que se sobreponga al anterior en términos de más completo, sino que implica el rodeo (die Umweg), es decir, el «no-camino», en el que el avanzar queda entorpecido y el único movimiento posible es profundizar; así entendemos la afirmación de Heidegger, según la cual no hay oposición entre objetivo-subjetivo como validación de procesos: no simple descripción orientada al objeto ni tampoco efusión de sentimientos. Esto nos da la dirección de la comprensión.

Así la direccionalidad coordina con los medios: lo que es propiamente discurso o aquello de lo que se habla, lo que realmente está ahí; se trata del presente de Agustín (deja atrás su pasado): "Lo que soy en el mismo momento de mis Confesiones" (Heidegger, 1995: 177); la declarada contemporaneidad implica una aproximación y un perfeccionamiento, que Heidegger lee como conexión entre el signo de la temporalidad agustiniana y el horizonte del sentido; sin duda, éste es un rastro que nos muestra con claridad el recorrido de la lectura de Heidegger, pues como dijimos, no traduce, sino que hace sucesivas compenetraciones del texto: sacar a luz la facticidad de la vida de san Agustín y mostrar así el «vivir en el mundo».

La ejecución de la comprensión: la alabanza (uno de los sentidos de «confesión») al Dios justo y bueno en la literalidad del ser dado al mundo y a su comprensión; de ahí la glosa del giro agustiniano ya

terim quod ad me attinet, hoc in me egerunt cum scriberentur et agunt cum leguntur. Quid de illis alii sentiant, ipsi viderint; multis tamen fratribus eos multum placuisse et placere scio. A primo usque ad decimum de me scripti sunt, in tribus ceteris de Scripturis sanctis, ab eo quod scriptum est: In principio fecit Deus caelum et terram $(\operatorname{Gen} 1,1)$, usque ad sabbati requiem (Gen. 1, 2)".

5 "Confessionum mearum libri tredecim, et de malis et de bonis meis [in meinem bösen und guten Sein, Leben, Gewesensein] Deum laudant iustum et bonum..." 
citado: "tanto por mis obras malas como por las buenas [en mi ser, en mi vivir, en mi haber sido malas y buenas], alaban a Dios justo y bueno" ("et de malis et de bonis meis [in meinem bösen und guten Sein, Leben, Gewesensein]"; las instancias del ser, del vivir, del haber sido malos o buenos recuperan la identidad y la unidad de la confesión, que se dirige a un Dios de perfección: "justo y bueno" [iustum et bonum]; nos queda, en el análisis, a contraluz de la vida divina, la pertenencia a la vida concreta, la profusión de su sentido en el tiempo (Heidegger, 1995: 177).

Aquí queda claro que no hay otra forma de poner atención en el ser humano que no sea histórica; en efecto, la historia personal no se opone a la perfección de Dios, en tanto dispone de su libertad; ésta es la vía que abre propiamente a la interpretación. Esta interpretación -para ser tal-debe constituirse en los términos de una decisión existencial.

Este acto esencial es el que conduce desde la subjetividad a la ejecución de la comprensión, pues al ser ejercida contiene y sobrepasa la visión objetivante, que no podía ser solo dejada de lado sino, en lo fundamental, asumida. Al hacer esto, funda su giro sobre sí misma y, por ello, la facticidad de la vida de san Agustín no es una tautología subjetiva (el riesgo permanente del interiorismo y de la interpretación de éste) sino auto-presencia originaria o unidad de la conciencia en acto ("tanto por mis obras malas como por las buenas").

La introducción a la comprensión del Libro X tiene cuatro momentos: a) el encuadre general del «confesar» (confiteri) y su significado; b) el saber de sí mismo; c) la objetualidad de Dios; d) la esencia del alma.

¿Qué sentido tiene confesarse ante Dios, que en su infinita sabiduría todo lo sabe?

En principio, la confesión de Dios, en el sentido que conlleva el genitivo subjetivo, expresa un detenimiento o pausa e implica que la subjetividad está siempre medida por la conciencia de sí; en este sentido, subjetividad significa simplemente la aptitud para abrirse a la posibilidad de captación, como una conciencia que no siempre dispone de sí; por ello, al ponerse ante Dios, no la niega, sino que la 
hace patente ante sí como subjetividad consciente. Se implica, entonces, una objetivación, en tanto se da en los términos de un hecho que define una determinada intimidad subjetiva-objetiva.

¿Qué significa confesarse ante el prójimo? En principio, un informe, [ein Bericht], sobre el ser ahora, sobre la actualidad de san Agustín, es decir, “dar cuenta de sí o confesar lo que sabe de sí mismo" (Heidegger, 1995: 178).

La primera fase de la confesión es "Me convertí en un problema para mí mismo” (Conf. IV, 4, 9) [“Quaestio mihi factus sum”] o captación de lo humano; se trata del ir a tientas de la autoconciencia subjetiva, inadecuada respecto de sí misma y, por ello, percibida como problema o percepción nítida del despliegue dificultoso propio de la existencia.

En otras palabras, se trata de la intimidad más profunda - hasta aquí- de la subjetividad: un cierto «modo en que soy», que sobrevuela los contenidos de la experiencia; en este agudizamiento sobre el mundo de la propia existencia se halla una tendencia originaria de la vida fáctica (Vélez López, 2014: 37).

Se abre camino así a la "tierra de la dificultad" que Heidegger coordina con “¿Qué amo, entonces, cuando te amo? (Conf. X: 6, 8) [Quid autem amo, cum te amo?]. Pasamos, entonces, a confrontar al yo en su captación de lo divino. Si bien la hermenéutica de Heidegger pasa por los contenidos de aquello que vive en Dios y que, en cuanto tal, es amado por el yo, se detiene breve, pero significativamente, en la conjunción temporal «cuando» (cum).

En efecto, si la primera persona del presente de indicativo pone en juego la experiencia de Dios (en el texto latino, san Agustín hace constante referencia a los contenidos de ver y oír respecto de Dios), le corresponde a la finitud la alabanza: "y sólo desde ahí, en el «cuando» anuncia el cielo y la tierra la alabanza de Dios, no ciertamente cuando mi disposición es la de quien está investigando científica y naturalmente" (Heidegger, 1995: 179).

La alabanza de Dios es justamente lo inverso de buscar inquisitivamente en la naturaleza. En efecto, el trascender requiere una determinada instancia de no-ser, es decir, el paso hacia la trascendencia; la limitación de la subjetividad radica en su finitud de ser. 
Sin embargo, no resulta suficiente la limitación para trascender; debe también tener conciencia de esa limitación: la alabanza de Dios ocupa ese lugar en la interpretación de Heidegger, en tanto pone a Dios como lo absoluto que se confiesa. En esencia, este acto de trascender reclama que el yo carezca completamente de aquello que se alaba.

Por ello, la atención de Heidegger se dirige a la unidad que se genera entre ambos seres: uno finito e intencionalmente encaminado al segundo como su término (pasar por el mundo físico y prescindir de él) y el otro, el reconocimiento de Aquel que nos hizo.

Heidegger sigue el recorrido de san Agustín de lo exterior a lo interior, en la formulación de la pregunta «¿Qué debe inquirir?» Pero más aún en la respuesta que da con la cita de Agustín («lo mejor es interior»): “¡Preguntar siempre un «cómo»! Bajo un «cómo»"(Heidegger, 1995: 180, n. 11). ${ }^{6}$

Si la pregunta está siempre, aunque cada vez de una manera distinta, bajo un «cómo» y si la realidad es todo lo que no es un yo, esto significa que aquello que no es un yo no radica tanto en una determinada vivencia de lo otro, sino -más profundamente- en la diferenciación reflexiva que elabora la subjetividad en su finitud.

Por ello, el correlato entre la superioridad de lo que es interior y el preguntar en el cómo («bajo» [unter] = lo que subyace al preguntar mismo de la pregunta, lo que no se reconoce como tal en lo que denominamos, en el párrafo anterior, diferenciación reflexiva) se configura en la infinitud de su expresión y se confirma en las formas negativas o sub-yacentes de lo que se calla en lo que se expresa. La subjetividad pasa a ser, entonces, lo no limitado, lo que puede ser todas las cosas.

No se trata, claro está, de una lectura de signo aristotélico, cuya atención esté puesta principalmente en las operaciones cognoscitivas, sino que proporciona la contingencia de la subjetividad que se explicita en el significado de su expresión.

Como anota Heidegger (1995: 180), no hay un haber sido creado objetivo y luego la nueva dirección de la investigación, que el

6 "('melius quod interius'): Immer ein 'Wie' fragen! unter einem 'Wie'". 
autor presenta como «desplazamiento de la pregunta» [die Verschiebung der Frage]: tal desplazamiento se produce bajo la presión de los fenómenos, lo que literalmente significa para Heidegger: "ya no si Dios es esto o aquello, sino si yo «en ello» $=$ «con ello» $=$ «viviendo en ello» encuentro a Dios" (Heidegger, 1995: 181). ${ }^{7}$

¿En qué consiste, para Heidegger, este desplazamiento de la pregunta? En principio, toma la primera distancia de lo que más arriba ha denominado la tradición griega afincada en el cristianismo; de ahí el problema de la objetivación también antes mencionado. Solo referimos lo obvio: encontrar a Dios es saberse viviendo en Él.

Pero tratemos de avanzar en sus consecuencias. En principio, importa comprender el giro de la cuestión: la concepción agustiniana abandona las distintas experiencias lúcidamente descriptas y comprendidas en favor de una objetivación que se connota en la noción de evidente.

En efecto, aquella noción de lo evidente solo puede aplicarse de derecho a lo que se plantea formalmente como objeto de conocimiento. Heidegger está dando el paso inverso en su lectura: lo evidente es el vidente en cuanto tal.

Si la auto-evidencia de la subjetividad resulta, en definitiva, su propia evidencia, entonces, su sentido no es hallable en la reflexión ni en los procesos de conocimiento. Solo cabe presentarla vivencialmente como tautología no objetivable.

¿Hasta qué punto se puede mentar tal inmediatez? En principio, una tautología no objetivable no requiere demostración alguna y, por ello, la afirma un juicio inmediato. La tautología que se pone de manifiesto en estos actos, no es ella misma un juicio, pero - esto es lo relevante- está conectada a todos los juicios.

Esto pone en marcha el camino de / hacia la memoria. El ponerse de camino [progredi] hacia la memoria debe estar acompañado por la certidumbre de que esta aproximación está determinada por las relaciones psicológicas que, en cuanto tales, es decir, en cuanto

7 "... nicht mehr, ob dies und das Gott ist, sondern ob ich "darin" $=$ "damit" $=$ "darin lebend" Gott finde". 
hábitos de selección y de clasificación, resultan transmitidos por tradición (Heidegger, 1995: 181).

De ahí la pregunta sintética de Heidegger: “¿cómo abrirse paso y acceder a otra dación de sentido?” (1995:181) acerca de cómo quebrantar la taxonomía esclerotizada y hallar otro entendimiento, que sea nuevo (con el sentido aquí elidido de «viviente y verdadero» por contraposición a la gama de significados del adjetivo «heredado» (überkommen).

Los momentos de la interpretación: el asombro ante la memoria

En la interpretación que sigue de la memoria, Heidegger intenta un primer paso hacia la superación existencial del orden de las relaciones y del sistema de referencias objetivo de la psicología. En este punto no nos detendremos fundamentalmente sobre la síntesis del texto de Confessiones sino, antes bien, en los comentarios que Heidegger suele realizar y colocar entre paréntesis, luego de una cita o de un análisis.

Lo primero que en el comentario se señala es la falta de continuidad de la exposición de san Agustín: no hay un todo ordenado linealmente, sino giros y retrocesos, que buscan ahondar los «enigmas de ejecución»(Vollzugsrätseln) y traer a cuenta contenidos que no habían sido tratados (Heidegger, 1995: 182).

En este sentido, Heidegger coloca entre paréntesis, luego de haber referido la dirección de san Agustín, lo siguiente: "Lo que Agustín trae a colación como fenómenos concretos desborda, desde el punto de vista del puro contenido, y atendiendo sobre todo a cómo explica los fenómenos, en qué nexos y determinaciones fundamentales-como, por ejemplo, beata vita-, el marco y la estructura del concepto usual" (1995: 182). ${ }^{8}$

El marco y la estructura del concepto usual [üblichen] quedan desbordados por el texto de san Agustín; esto es, precisamente, lo

8, (Was Augustin an konkreten Phänomenen beibringt, rein inhaltlich, und vor allem wie er die Phänomene expliziert, in welchen Grundzusammenhängen und -bestimmungen -z. B. beata vita-, sprengt den Rahmen und die Struktur des üblichen Begriffes)." 
que interesa a Heidegger. La noción de vida feliz constituye el ejemplo referenciado. Intentemos entender el motivo de ello.

La vida en cuanto feliz realiza su propio acto de conciencia y por ello resulta particularmente activa; la facticidad del yo es radical, pues se pone a sí mismo en el primer plano del acto de conciencia. En otros términos: el yo se encuentra siendo. Surge así la vida como verdad absoluta del yo, es decir, la aptitud de la subjetividad para tenerse a sí misma.

Este nivel, quizá el más hondo, el yo mismo constituido en sí, radica agustinianamente, en vida feliz, pues la subjetividad está en su propia conciencia y, por ello, es un hecho (en el sentido latino de factum), es decir, algo que no es pura objetividad, sino fundamentalmente un hacer de su ser un hecho puro.

Así se presenta san Agustín a Heidegger y por ello desborda la cota de los conceptos usuales: la continuidad inasible de un momento es más que la explicación conceptual.

Desde el momento que este proceso se transforma en «algo pensado» [cogitatum] que tiene la voluntad de esencializarlo, desaparece para esa subjetividad lo irreductible del «ser pensante» [cogitans] y queda, como remanente, un pensamiento pensado, objetivado.

“y (los hombres) se olvidan de sí mismos...” [...et (homines) relinquunt seipsos] cita Heidegger (1995: 182) a san Agustín, lo que significa que no se admiran de que hablan de ello (las montañas, el mar, los ríos, los astros), porque de algún modo los habita interiormente; por esta razón, el lector desprevenido queda desorientado por san Agustín al momento de realizar una consideración demasiado detallada de la memoria. Por eso, ha dicho antes el Hiponense -también citado por Heidegger-: "La admiración [stupor] se apodera de mí" (1995: 182).

¿Cómo llegan los objetos no sensibles a la conciencia (ins Bewußtsein)? (Heidegger, 1995: 184). En esta pregunta no está mentado el proceso en sí, sino su resultado en la memoria. Por el contrario, al reducir los hechos de conciencia al producto de la materia se dice, por un lado, que están ligados a procesos fisiológicos, y que, por otro, son algo diverso de hechos únicamente fisiológicos. 
Complementariamente san Agustín se detiene en el correlato de la memoria (o «tener disponible para»: praesto), es decir, el olvido (oblivio); Heidegger calibra detenidamente la expresión agustiniana: "La memoria por la que recuerdo, el olvido que recuerdo" (Memoria qua meminerim, oblivio quam meminerim) (Heidegger, 1995: 188).

Primeramente se siente obligado a defender el modo de expresión, que podría considerarse un barroquismo: no se trata ni de un juego de palabras ni de sutilezas retóricas; por el contrario -acota Heidegger- el problema está planteado tan agudamente como es posible (Heidegger, 1995: 188).

Heidegger considera entonces que praesto est («tener como disponible» ha glosado poco antes) referencia -todavía de manera indiferenciada - el contenido y la ejecución de la representación (Vergegenwärtigungsvollzug) de memoria y olvido; por ello, el problema se mantiene en reconocer esta co-presencia, en tanto mantiene en pie la aporía, dado que no pueden convertirse mutuamente y estar presentes al mismo tiempo: para los que viven el olvido es posible mantenerse sin representación de ello.

Queda claro que, en esta circunstancia, la pregunta sobre qué es la memoria no es adecuada, aunque sí tiene un sentido preparatorio; el verdadero problema, el que es poco factible hallar con este modo de preguntar, tiene que ver con el movimiento de la memoria, lo que Heidegger intenta determinar como el contenido y como la ejecución de la representación.

Si la memoria se mueve en la existencia humana, lo que es concretamente, no debe buscarse en ella misma; su propósito, en efecto, está en su propio existir.

Con la finalidad de desocultar aquellas estructuras, constituye tres niveles de comprensión: a) el sentido referencial (Bezugssinn), b) su sentido de ejecución (Vollzugssinn), y c) el respecto de qué, la referencia o sentido de contenido (Gehaltssinn). El sentido de ejecución tiene un significado especial pues, a diferencia de la nóesis husserliana, no pone el acento únicamente en la forma cognoscitiva del acto.

La ejecución (Vollzug) es una expresión que connota cómo se realiza la vida, sin reducirla a los actos de conocimiento, aunque tampoco excluyéndolos. El sentido de ejecución (Vollzugssinn) tiene 
una primacía sobre los demás en la medida que determina de qué modo concreto se realiza la referencia al objeto y cómo éste se hace presente. Los objetos no tienen un sentido independiente del modo como nos relacionamos o nos comportamos hacia ellos (De la Maza, 2005: 138).

Heidegger deja abierto el espacio sobre la aporía del olvido (oblivio) con la siguiente cita de san Agustín y dos glosas: "Y entonces (...) estoy seguro de que recuerdo el mismo olvido [«El tener olvidado»], mediante el cual se oculta lo que recordamos [«Lo que queremos tener presente»]" (Heidegger, 1995, 189). ${ }^{9}$

¿Qué se gana con la diversificación de la pregunta? En principio hay así una interconexión fenomenológica entre «amar»/ «buscar»y la representación de los modos subjetivos de estar ante las cosas, que tiende a hacer comprensible la objetividad en sentido óntico.

Esto sucede por la misma validez de la subjetividad, cada vez que es también considerada como fenómeno; ello implica que la investigación (el buscar de Heidegger) tiene la dirección de cada uno de los modos en que aquella subjetividad está dada.

Lo que denominamos vivencia es, en realidad, un conjunto de vivencias en las que se distingue sucesivamente un antes y un después, pero en la unidad de la corriente. Es el espacio del mundo vital, tal como lo encontramos en Husserl (el vivir la actitud vital).

En este propósito de volver relativo todo intento de objetivismo, la subjetividad se comprende a sí misma como «presencia en sí», como el modo de percibirse ahí. Si consideramos temporalmente esta relación, hablamos del instante.

Por ello, ¿qué amo? o ¿qué busco? tiene una respuesta inmediata en san Agustín: la vida feliz. La dirección del buscar se sostiene ahora en una teoría general del acceso (Heidegger, 1995: 193).

¿A dónde conduce el acceso (der Zugang)? A concretar la pregunta sobre cómo se ha de buscar a Dios; por ello, la pregunta ha mutado del amar (amare) al buscar (quaerere); «mutar» significa transformarse manteniendo el primer estado, pues el amar está en el buscar

9 "Et tamen [. . . i ipsam oblivionem [das Vergessenhaben] meminisse me certus sum, qua id quod meminerimus [was wir vergegenwärtigen wollen] obruitur. 
y viceversa, en especial si el objeto es Dios. El centro del discurso retoma la noción de ejecución (derVollzug).

Este giro implica que Heidegger ha puesto en relación el pasaje ya citado de Conf. $(\mathrm{X}, 6,8)$ con otro del mismo libro, que abre el camino al giro que aludimos; nos referimos a $\mathrm{X}, 29$ : "Cuando te busco, Dios mío, busco la vida feliz. Te busco para que viva mi alma” (Heidegger, 1995: 192-193). ${ }^{10}$

En este sentido, Costantino Esposito (2006: 60) señala que buscar a Dios significa buscar la vida y buscar la vida tiene el significado de una preocupación en torno a la vida, es decir, aquella inquietud que es la vida misma como angustia.

En el cruce de textos que mencionamos, Heidegger recobra el significado de la vida feliz (beata vita) a partir de la experiencia del gozo (gaudium); se pone de manifiesto aquí un cambio profundo en la elucidación de lo que significa experimentar: no alcanzar un conocimiento, sino una identificación en el núcleo del sujeto en quien se da la experiencia. Así explica Heidegger la condición de expertus, de persona experimentada, que reclama para sí san Agustín (Conf. X, 30), en tanto disfruta de su yo.

Si la vita beata se reconoce en el regocijo del que percibe tal experiencia, entonces, se transforma en el camino de la alegría [“in der Weise der Freude"] y el modo en el que existe: "El deleite es el fin de la preocupación” (“delectatio finis curae”) (Heidegger, 1995: 196). Por ello, el estado de tristeza actual no es impedimento para cesar la actualidad de la alegría, pues la vida feliz [vita beata] no se circunscribe al feliz [beatus], sino que también incluye al desgraciado [miser] en cuanto es el tono en el que existe el existente.

Heidegger se preocupa en dejar claro que no se refiere a estados de ánimo en el sentido psicológico de la expresión. En efecto, si la felicidad es algo que está disponible (verfügbar), como el ambiente propio del Dasein y como lo que constituye significativamente su mundo, ésta le es accesible (erreichbar) (Heidegger, 1995: 196).

10 "Cum enim te, Deum meum, quaero, vitam beatam quaero; quaeram te, ut vivat anima mea". 
Ésta es la formalidad de la felicidad; lo que importa, sin embargo, es cómo se la apropia cada uno, pues en ese momento es real para cada uno. En este sentido, el estado de ánimo implica que el encuentro con la vida feliz no se limita necesariamente a ella, sino que se explica como toma de conciencia de ella.

El horizonte de la consideración se transforma en estos términos: si la felicidad es accesible en cuanto constituye su mundo como un giro sobre sí mismo, entonces, y de modo enfático, aquella es tautológica y heterológica (el yo sale al encuentro de algo que no se reduce a él).

El correlato feliz/desgraciado (beatus/miser) significa, en el presente contexto, que el Dasein conserva sus límites al referirse a estas vivencias reflexivamente, en tanto que objetivarlas resulta, en un sentido concreto, desposeerlas de su carácter originario de vivencia.

La relación de copertenencia previa a la objetivación es necesariamente imprecisa, porque es la posición del Dasein ante la totalidad del ser; es evidente que imprecisión no tiene aquí un sentido peyorativo o descalificador, sino que expresa la posición primigenia del Dasein frente al todo como todo. Para que se pudiera sostener un sentido descalificador de imprecisión se requeriría que lo puesto en consideración fuese claramente determinado en su entidad.

La vivencia originaria de quien sale al encuentro de la verdad se expresa en términos de alegría: allí donde la vida ha salido a nuestro encuentro, justamente allí experimentamos su verdad. En estas experiencias, en las que no queremos ser engañados, se produce el encuentro con la verdad.

Si se acepta que un modo de hablar es un modo de pensar, entonces, esta alegría nunca llega a un modo de «no ser», en el sentido de «no ser ya». La verdad que encuentra la vida feliz es la propia conciencia de sí y de Dios -"la vida de la vida” (VI, 10)-. Por ello, el recurso de la alegría le otorga al itinerario un dinamismo que no es de rígida unidad, sino de una corriente que vuelve al Otro y lo descubre como manantial.

Éste parece el sentido más intenso de la lectura para Heidegger: la memoria coloca al hombre frente a su propia existencia, haciendo un infinito del horizonte de posibilidad. Se vincula así la memoria, 
que rescata la existencia de su propio fluir y que implica la idea de un tiempo originario, con la felicidad que goza de la verdad y de la propia verdad. La presentación que san Agustín hace de memoria y de gozo y la personalísima lectura de Heidegger abren una nueva forma de entender el tiempo (Dias, 2011: 71).

Sin embargo, hay una fragilidad intrínseca de la vida y de la memoria: la verdad puede caducar o tomar una dirección descendente, (in der Abfallsrichtung) (Heidegger, 1995: 199-201; Dahlstrom, 2010: 268). La alegría está acechada entre el ruido interior de la agitación y el alboroto, pero el tenue eco que se conserva en la memoria mantiene el reclamo de su existencia en relación a la verdad.

¿A qué se debe que la auténtica verdad no sea considerada sino odiada, si ésta está ahí naturalmente dada? La cita poco anterior de san Agustín dice: "Hay todavía un poco de luz [modicum lumen] en los hombres" (Conf. X, 23, 33) y luego la indicación sobre «un poco de luz»: tiene fundamentalmente un sentido de ejecución (Vollzugssinn) que se aplica a la experiencia fáctica, es decir, la dirección propia del experimentar (en el sentido que esto adquiere en la re-presentación de usar o considerar). Éste es el hilo conductor de su analítica de la facticidad, que, como tal, lo aleja de la consideración metafísica de la luz, pues se aplica al seguimiento de la vida en movimiento.

Todo fenómeno vital reside en un cierto impulso que lo delimita; tal delimitación comprende ese movimiento hacia la realidad que llamamos percepción. Implica un cierto estado de indigencia en comprender lo que es como tal; éste es el principio y el límite de la experiencia, que no pertenece propiamente a la esfera de la conciencia, en cuanto vivencia.

En razón de ello, el giro «mundo propio» (Selbstweltlich) (Heidegger, 1995: 219) es un modo de plantear la experiencia a partir de la actividad que implica la propia experiencia, antes que del contenido de conocimiento que pudiera expresar (Fischer, 2007: 338).

Consideramos que así se concentra el paso de la fenomenología a la hermenéutica, pues la pregunta sobre quién sobrelleva estos procesos posibilita el acceso al análisis de la experiencia vivida.

En la subjetividad como «ipseidad» echa raíces el dónde de la pregunta por el ser. El sentido de ejecución, al que nos referimos 
más arriba, se completa y acrecienta en la sugerencia de Heidegger sobre el sentido de la luz: hilo temático de una filosofía de la vida inaccesible a la metodología fenomenológica, con el sentido que ésta tiene en Husserl, porque la habita el problema del sujeto.

En efecto, no se trata de fijar y, consecuentemente, de describir términos que expresan lo que Heidegger llama objetivar, sino de determinar qué se da en la facticidad del tiempo; lo que se da no es otra cosa que un momento (caracterizado, por consiguiente, por la parcialidad y la precariedad), factible de ser reconstruido de manera retrospectiva al interior de su devenir perpetuo (la totalidad de aquel momento).

Si la fenomenología descansa en que «fenómeno» es «aquello que simplemente se da», entonces, este darse se cumple necesariamente en un contexto determinado por el sentido, es decir, por la confluencia de tendencias y motivaciones.

Se impone la pregunta sobre el sentido: ¿qué organiza o estructura el mundo en que vivimos? Esta pregunta se desliza sobre dos cuestiones que resultan inescindibles de ella: a)¿Cuál es el sentido de «origen»? y b)¿Cuál es el origen del sentido? Esta segunda es la que resulta definitiva en la interpretación que nos ocupa (Villevieille, 2013: 90; Rodríguez, 1997: 28).

La importancia (die Bedeutsamkeit) (Heidegger, 1995: 219) del conjunto de la pregunta radica en que el núcleo de articulación de lo que se da en el mundo es la vida, según la semántica helénica de vida (zoé). En este sentido concreto, la fenomenología de cuño husserliano va camino a la hermenéutica de la facticidad o manifestación de la experiencia desde la que se forma un concepto (León, 2009: 3-4).

Ancla aquí la radicalidad de la dimensión semántica, pues ésta delimita el contexto de la experiencia originaria. Así como no expresamos lo que es el ser, sino lo que pensamos de él, queda claro que la facticidad requiere de un lenguaje que represente de manera creativa y lo más vivencialmente posible los conceptos intuidos en la experiencia. Esta relación debemos comprenderla siempre de manera provisional, al ser susceptible de permanente profundización. 


\section{Conclusión}

En Agustín y el Neoplatonismo, Heidegger se ocupa de desmontar las interpretaciones que, desde distintos puntos de vista, objetivizan, es decir, abandonan a la irrealidad del desconocimiento su tema de estudio. Ello no implica retomar una senda subjetivizante, pues sería tan improductiva como la primera; en efecto, en ambos casos, queda oculta la trama de la vida efectivamente vivida. Este es el sentido de llevar adelante la crítica a los autores estudiados y poner en evidencia que, en esos términos, la vida queda fuera del conocimiento histórico.

Para alcanzar efectivamente este objetivo, el primer paso consiste en considerar «el sentido del acceso» (Zugangssinn), que abre la discusión de las tres concepciones presentadas en la primera parte del texto.

En efecto, queda claro que, desde la perspectiva de Heidegger, la historia del pensamiento presentada a partir de síntesis paradigmáticas no resulta representativa del acontecer histórico; así, por ejemplo, Agustín y el problema de la cultura cristiana (E. Troeltsch); Agustín y el descubrimiento de la piedad en el Mundo Antiguo (A. von Harnack) y, por último, Agustín y el fundamento de la realidad absoluta de la experiencia interior (W. Dilthey). Todas ellas expresan un planteo metodológico inadecuado, porque implican una completa incomprensión de lo que significa «objetividad histórica» (la distinción verdadero-falso tiene un lugar relativo en la comprensión de la historia).

Por ello, el joven Heidegger se aboca a la captación de las estructuras del ser como vida fáctica: éste es el objeto de la aplicación del modelo de lectura de Heidegger. En este sentido, la posibilidad de alcanzar este objetivo radica en la capacidad de distinguir las categorías conceptuales que constituyen las diversas interpretaciones culturales y experienciales.

Realizada esta primera precisión, plantea la ejecución del acceso (Zugangsvollzug), que consiste en echar raíces en la vida; la ejecución (Vollzug), en efecto, es el punto de partida de la comprensión; ello implica que la realidad es resultado de aquella efectuación y que ésta tiene un claro sentido semántico. 
Es aquí donde se define la distancia con la metafísica tradicional, pues ésta descansa en la seguridad de poder descubrir lo constitutivo de la realidad mediante el lenguaje. Por el contrario, en el sentido que Heidegger plantea la ejecución (der Vollzug), la operación está sostenida en la semántica que el proceso implica. Esto significa, por un lado, que, en el caso que nos ocupa de san Agustín, el acceso a la vida fáctica es textual y, por otro, que la puesta en marcha o ejecución del proceso consiste en interpretaciones que organizan un horizonte de sentido.

Esta dirección tiene dos pasos esenciales: por un lado, el comentario (Das Referat), que no es propiamente un texto más completo en cuanto al sentido, sino un restablecer para sí la comprensión del texto. Por otro, las glosas o paréntesis en los que Heidegger reescribe en clave propia el sentido del latín de san Agustín (recordemos que lo declaró intraducible a causa de su perfección).

Este modo de comentario no implica avanzar en la clarificación del verdadero sentido sino, por el contrario, un cierto detenerse en el «no-camino» (die Umweg), donde avanzar queda entorpecido. En consecuencia, el único movimiento posible es profundizar; así entendemos la afirmación de Heidegger de que no hay oposición entre objetivo-subjetivo como validación de procesos: no simple descripción orientada al objeto, ni tampoco efusión de sentimientos. Así percibimos también la dirección de la comprensión.

El tema y el método fueron tratados simultáneamente por el joven Heidegger; el método queda definido por la vida fáctica. Por ello, una y otra vez surgen los intentos por responder la misma pregunta: ¿cómo es posible aprehender el fenómeno de la vida sin hacer uso de las herramientas intelectuales objetivantes de la tradición griega asumida y transformada por el cristianismo?

Podemos concentrar los distintos accesos que dimos como respuesta, en el presente trabajo, en estos términos: si el ser de los entes, considerada esta expresión en sede fenomenológica, es alcanzable como fenómeno, significa que éste se muestra en su «cómo», al que se accede por vía hermenéutica. Hemos señalado antes que la tarea de interpretar no es especificar algo en tanto comprendido, sino abrir máximamente las posibilidades de comprender. 
Nuestro aporte consiste en haber señalado que, en la lectura que Heidegger hace de san Agustín, comienza a mostrarse el camino de la hermenéutica en cuanto permanente ejercicio de auto-interpretación del estado de cosas, al que se accede en la lectura de aquel «cómo».

Aquí encontramos claramente subyacentes las intuiciones fundamentales de las estructuras del Dasein y, con nitidez, desde el punto de vista del método, la fenomenología que transita hacia la hermenéutica en el ejercicio del «no camino» [die Umweg], uno de los modos de estar en relación con el mundo.

\section{Referencias bibliográficas}

\section{Fuentes}

Heidegger, M. 1995. „Augustinus und der Neuplatonismus“. En Phänomenologie des religiösen Lebens, GA 60, 160-269. T. Regehly, C. Strube, M. Jung (hrsg.). Frankfurt am Main: Vittorio Klostermann.

“Agustín y el neoplatonismo”. (1997). Fenomenología de la vida religiosa, 23-210. J. Muñoz (trad.). Madrid: Siruela.

“Agostino e il Neoplatonismo". (2003). Fenomenología della vita religiosa. G. Gurisatti (trad.). Milano: Adelphi.

"Augustine and Neo-Platonism". (2004). The Phenomenology of Religious Life, 115-226. M. Fritsch, J. A. Gosetti-Ferencei (trad.). Bloomington: Indiana University Press.

.Augustin et le Néoplatonisme”. (2011). En Phénoménologie de la vie religieuse, 178-344. J. Greisch (trad.). Paris: Éditions Gallimard.

\section{Repertorio}

Camilleri, S. (2008). Phénoménologie de la religion et herméneutique théologique dans la pensée du jeune Heidegger: Commentaire analytique des fondements philosophiques de la mystique médiévale (1916-1919). Dordrecht: Springer Netherlands.

Campbell, S.M. (2012). The Early Heidegger's Philosophy of Life. Facticity, Being and Language. New York: Fordham University Press. 
Caputo, J.D. (1999). "Heidegger and theology". En Ch. B. Guignon (ed.). The Cambridge Companion to Heidegger, 270-288. New York: Cambridge University Press.

Dahlstrom, D.O. (2010). "Truth and Temptation: Confessions and Existential Analysis”. En SJ. Grath, A. Wiercinski (eds.). A Companion to Heidegger's Phenomenology of Religious Life, 263-284. Amsterdam / New York: Rodopi.

De la Maza, L.M. (2005). "Fundamentos de la Filosofía Hermenéutica: Heidegger y Gadamer”. En Teología y Vida, XLVI: 122-138.

Dias Sousa Ferro, B. (10 octubre, 2016). Heidegger leitor de Agostinho: A memória como fenómeno existencial. Faculdade de Ciências Sociais e Humanas, Lisboa, Universidade Nova de Lisboa. Obtenido en: https: / /run.unl.pt/

Escudero, J.A. (10 octubre, 2016). El programa filosófico del joven Heidegger. Introducción, notas aclaratorias y glosario terminológico sobre el tratado "El concepto de tiempo". Barcelona: Herder. Obtenido en: www.herdereditorial.com > Filosofía > Ensayos.

Esposito, C. (2011). "Heidegger, von der Faktizität der Religion zur Religion der Faktizität" N. Fischer-FW. von Herrmann (hg.). Die Gottesfrage im Denken Martin Heideggers: 47-68. Hamburg: Meiner Verlag.

Fischer, M. (2013). Religiöse Erfahrung in der Phänomenologie des frühren Heidegger. Göttingen: Vandenhoeeck \& Ruprescht.

Flamarique, L. (2013). "Practicar la verdad: Sintonías y disonancias de Heidegger con el libro X de Confesiones”. Tópicos, 44: 115-148.

Held K. (1995). “Edmund Husserl”. En O. Höffe. Klassiker der Philosophie.Von Immanuel Kant bis Jean Paul Sartre, II: 274-297. München: Verlag Beck.

León, EA. (10 octubre, 2016). "El giro hermenéutico de la fenomenología en Martín Heidegger”. Polis, 8 (22): 267-283. Obtenido en: http: / bit.ly/1bf80q9

Rodríguez, R. (1997). La transformación hermenéutica de la fenomenología. Una interpretación de la obra temprana de Heidegger. Madrid: Tecnos.

Stanley, T. (2010). Protestant Metaphysics after Karl Barth and Martin Heidegger. Eugene (Oregon): Cascade Books.

Toadvine, T, L. Embeec (eds.). (2002). Merleau Ponty's Reading of Husserl. Dordrecht: Kluwer Academic Publishers.

Vélez López, G.D. (2014). Vida y Filosofía: la génesis de la vida filosófica en Heidegger. Bogotá: Fondo Editorial EAFIT.

Villevieille, L. (2013). "Heidegger, de l'indication formelle à l'existence". En Bulletin d'analyse phénoménologique IX, 5 : 1-96. 